\title{
Non-surgical follow-up success in blunt abdominal trauma. Can we protect patients with blunt abdominal trauma from surgery?
}

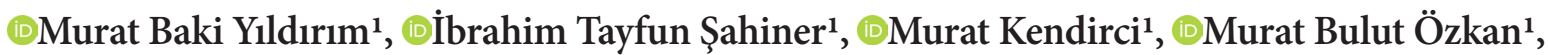 \\ ๑Durathan Erkent ${ }^{2}$, ๑Ramazan Topçu ${ }^{1}$, @Settar Bostanoğlu ${ }^{1}$ \\ ${ }^{1}$ Hitit University' Faculty of Medicine, Department of General Surgery, Çorum, Turkey \\ ${ }^{2}$ Başkent University' Faculty of Medicine, Department of General Surgery, Ankara, Turkey
}

Cite this article as: Yıldırım MB, Şahiner İT, Kendirci M, et al. Non-surgical follow-up success in blunt abdominal trauma. Can we protect patients with blunt abdominal trauma from surgery?. J Health Sci Med 2021; 4(3): 295-299.

\begin{abstract}
Backgrounds: Abdominal traumas have an important place in the emergency room. Historically, surgical intervention was adopted as a treatment method for liver and spleen traumas. However, with the development of imaging methods, the possibility of non-surgical follow-up was obtained. In this study, the advantages and disadvantages of conservative treatment for surgical intervention were investigated.

Material and Method: The patients with complaints of blunt abdominal trauma, who were admitted to the third step research center emergency service during the 27-month period and were referred to general surgery, were retrospectively examined.

Results: Of patients, $58.2 \%(n=53)$ was monitored conservatively, $34.1 \%(n=31)$ had splenectomy and $4.4 \%(n=4)$ underwent the primary repair due to isolated liver injury, and both splenectomy and liver primary repair were performed in $3.3 \%(n=3)$ because of multiple solid organ injury. We observed that the injuries of non-surgical spleen trauma patients changed between grade I and III. All patients with grade IV-V injuries underwent splenectomy. Patients with decreased hematocrit and whose hematocrit did not increase despite erythrocyte replacement were operated on.

Conclusion: In this study, it was explained that patients who were followed up without surgery did not need surgery and that both liver trauma and spleen trauma should be given the chance to follow up without surgery.
\end{abstract}

Keywords: Blunt trauma, liver, spleen, non-operatif,

\section{INTRODUCTION}

Traumas constitute an important part of emergency service admissions. In developing countries, traumas are among the main causes of mortality and morbidity under the age of 45 . The abdomen is the third most frequently injured part of the body (1). Approximately $7.5 \%$ of trauma patients in Turkey are treated in the general surgery department (2). The general state of the patient should be taken into account when evaluating abdominal traumas. The abdomen has a large number of organs, which have different characteristics and are mobile or stationary (3). The most injured solid organs in blunt abdominal trauma are the spleen and liver (4). According to physical examination and imaging performed in the emergency department, patients who have the necessary surgery must be diagnosed quickly and should be operated.
Historically, most of the abdominal traumas have been operated, however, the development of imaging methods and patient follow-up opportunities increase the view of non-surgical follow-up. Non-operative Treatment (NOT) is standard in patients who have a non-acute blunt hepatic injury and are hemodynamically stable. Successful NOT may provide the low need for transfusion, low infection rate, can reduce hospital stay duration and have a positive effect on survival in hemodynamically stable patients with high-grade liver injuries (5). However, it has been reported that the chances of success are lower when there is a spleen trauma or kidney injury accompanying liver injuries (6).

In this study, the frequency of abdominal organs injury, conservative approach and surgical outcomes of patients admitted to the emergency department with blunt abdominal trauma were reported. 


\section{MATERIAL AND METHOD}

\section{Ethical Approval}

The study was approved by The Hitit University Medical Faculty Erol Olçok Training and Research Hospital Non-interventional Research Ethics Committee (Date: 26.10.2018, Decision No: 2018-180). Informed consent was received from all patients. All procedures were performed adhered to the ethical rules and the principles of the Declaration of Helsinki.

\section{Patients}

The patients who applied to the emergency department of Hitit University Medical Faculty Erol Olçok Training and Research Hospital on 01.01.2016-01.03.2018 due to blunt abdominal injury were retrospectively evaluated. Records for all patients were obtained from their files. Patients who we could not access the sufficient information on and the cross-sectional imaging examinations from the files were excluded. Patients with suspected hollow organ injuries were excluded from the study. Demographic characteristics, cross-sectional examinations, which organs were injured, surgery type, duration of hospital stay, mortality and morbidity of the patients were evaluated.

\section{Patient Monitoring}

Conservative treatment was performed for abdominal injuries of patients who were hemodynamically stable without any signs of peritonitis. The patients underwent surgery when their hemodynamics disappeared or when there was a deterioration in the clinical course.

\section{Statistical Analysis}

SPSS 20.0 program was used for statistical data. The data were recorded as percentage, mean, frequency and standard deviation.

\section{RESULTS}

\section{General Findings}

In the 27-month period, a total of 690 patients with blunt abdominal trauma applied to the emergency room and were referred to our clinic. Ninety-one of the patients were hospitalized and treated. All of these patients were firstly evaluated by the consultant general surgeon and decided to be hospitalized. $39.6 \%$ of patients (n:36) is female, $60.4 \%$ (n:55) is male, and total average age is 45,4 . The majority of traumas were between $2^{\text {nd }}$ and $6^{\text {th }}$ decades.

\section{Initial Evaluation}

During the cross-sectional examination made in the emergency room, it was observed that $29.7 \%$ ( $\mathrm{n}: 29$ ) had a general body trauma, 39.6\% (n:36) had a spleen laceration, 9.9\% (n:9) had a liver laceration, 7.7\% (n:7) had a liver and spleen laceration, and 13.2\% (n:12) had an intraabdominal free fluid, however any solid organ damage that accompanied was not detected. In addition, the hematocrit values at the time of admission and the control hematocrit values at the 4 th hour were evaluated.

\section{Clinical Course}

When the clinical course of the patients was examined, it was observed that $58.2 \%$ (n: 53 ) of the patients was conservatively followed up, $34.1 \%$ (n: 31 ) had splenectomy, $\% 4.4$ (n:4) underwent primary repair due to isolated liver injury, 3.3\% (n:3) underwent both splenectomy and liver primary repair due to multiple solid organs injury. Patients with general trauma (n:27) were all conservatively followed up and none of them had any surgical requirements. In the follow-up of these patients, hematocrit decline was an average of 1.52 units and no blood transfusions were performed. We observed that 9 patients with isolated spleen laceration were followed up without surgery, and 27 patients had a splenectomy. The average reduction of the mean hematocrit in the non-operated patients was 2.5 units and it was 8 units for the operated patients.

\section{Radiological Findings}

When cross-sectional imaging was examined, it was observed that there were 6 patients with grade I, 2 patients with grade II, and 1 patient with grade III in the nonsurgical follow-up group with isolated spleen trauma. The cross-sectional imaging methods of the patients with isolated spleen trauma were examined, there were 4 patients with grade II, 9 patients with grade III, 13 patients with grade IV and 2 patients with grade V laceration.

\section{Treatment Follow-up}

In this study, 7 of 9 patients who were detected to have liver laceration were followed as conservatively, whereas two patients underwent primary liver suturation. According to the distribution of these patients, 5 patients had grade I-II injuries and 4 patients had grade IV injuries. All patients with grade I-II injuries and 2 of the patients with grade IV injuries were followed up without surgery. The average hematocrit decline in patients with no surgery was 3.5 units, while the decline in operated patients was 5 units. In the imaging, multiple solid organ injuries were detected in 7 patients, 2 of them had an only splenectomy, 2 had liver repair, and the other 3 had both liver suturation and splenectomy due to liver and spleen injury. The hematocrit decline during the follow-up of these patients was 10 units. Patients, who were reported to have fluid only in the abdomen and had not a solid organ injury in the tomography, were examined, 10 of the patients were followed up conservatively, while 2 patients underwent the splenectomy. The mean decrease in hematocrit in these patients was 1 unit in the nonoperated followed group and 13.5 unit in the operated patient group. The mean duration of hospitalization was 6.9 (1-24) days. This period was approximately 5.7 days in patients who were followed up without surgery while was 8.5 in the patients with surgery. 


\section{DISCUSSION}

Historically, the operational methods have been preferred in all patients with abdominal trauma by looking at physical examination findings $(7,8)$. However, with the development of imaging technologies, non-operative methods have been preferred (9). Various studies report that patients can be followed up non-operatively when they are hemodynamically stable, regardless of the severity of the injury. However, in the case of deterioration of the general condition of these patients or if they need more than 5 units of blood transfusions within 24 hours, the preparations for emergency surgery should be made (10). Patients should be closely followed up hemodynamically and should be re-evaluated with cross-sectional imaging methods and ultrasound imaging as needed.

The most injured organ in patients with blunt abdominal trauma is the spleen (11). Historically, splenectomy has been the preferred method in most of the highgrade splenic injuries, however, surgeons have begun to prefer postoperative follow-up because of infectious complications in post-splenectomy. The American Association for the Surgery of Trauma developed a grading system for spleen injuries. The literature studies show that a significant mortality rate $(22.7 \%)$ is present in grade $\mathrm{V}$ laceration (12-14). This grading system assists surgeons in choosing the treatment method of the patient. In this study, 36 patients with isolated splenic laceration were present. $75 \%$ of these patients underwent splenectomy, and $25 \%$ of these patients were followed up non-operatively. Patients with non-surgical follow-up had spleen laceration between grade I-III. In the followup of these patients, an average decrease of 2.5 units of hematocrit was observed. However, this decline was not evaluated as important because there was no evidence for any acute bleeding. Therefore, the available data are important, and the experience of the team following the patient with blunt abdominal trauma is important in the interpretation of these data. Thus, it can protect patients from unnecessary surgery and from the possibility of a ruptured spleen.

According to the degree of injury of the operated patients, there were 4 patients with a grade II injury, 9 patients with grade III injuries, 13 patients with grade IV, and 2 patients with grade V. Patients with low-level injuries or being hemodynamically stable were followed up without surgery. However, it was observed that 6 patients with grade II-III injuries were hemodynamically unstable at the time of admission and 3 of them were operated because of free organ perforation. In addition, 4 patients with hypotension, with more than 10 units of hematocrit decline, and with grade II-III spleen laceration were operated, then splenectomy procedure was performed. Four patients with grade II-III injuries were operated due to hematocrit decline more than 15 units between the hematocrit values at the time of admission and the 4th-hour control results. Several studies have reported cases of delayed splenic rupture in patients followed up without surgery (15). In this study, splenectomy was applied to 4 patients, who were not given an operation decision at the time of admission, due to hematocrit decline and hemorrhagic bleeding detected through the imaging techniques. Non-surgical follow-up may shorten the duration of hospitalization of patients, but may require prolonged immobilization of the patient, may cause abscess of hematomas that may occur, and may cause delayed hemorrhages, therefore surgeons should be careful in the decision making $(15,16)$. Mortality and morbidity were not detected in the non-surgical follow-up group, while the number of deaths was 3 in the splenectomy group. However, we observed that these patients had a higher energy and non-abdominal injuries.

In blunt abdominal traumas, the second most frequently injured organ after the spleen is the liver. It is known to be injured at the rate of $1-8 \%$. In the past, surgery was recommended for all patients with liver injury, because the majority of abdominal traumas were caused by liver injuries (17). Liver injuries were graded by the American Association for the Surgery of Trauma. According to the severity of the injuries in the liver, it is divided into grades between I and VI (13). The two patients who underwent surgery had grade 4 lacerations but were found to be hemodynamically unstable at the time of admission. The failure rate of non-surgical follow-up in studies has been reported as $6 \%(18,19)$. In the present study, none of the patients who were followed up without surgery had no delayed surgical requirements. Recent studies have reported that the success rate of non-surgical follow-up in patients with high-grade liver injuries is between $60-70 \%$ $(20,21)$. In the present study, for 4 patients with Grade IV injuries, non-surgical follow up was achieved at the rate of $50 \%$. The reason for the low rate of non-surgical follow-up may be the absence of vascular embolization at the center of the study.

Although no solid organ damage is detected in imaging methods, patients with intra-abdominal free fluid should be closely followed up. These patients can have free abdominal fluid due to mesenteric vascular structures or solid organ damage (22-24). In this study, we observed that 2 of 12 patients, who did not have solid organ damage at the time of admission or did not show a significant decrease in hematocrit level, underwent splenectomy operation. These two patients had a grade- 4 splenic injury. Although the sensitivity and specificity of the computed tomography reach $80 \%$ in spleen injuries, it should be kept in mind that there may be injuries that imaging methods can miss in blunt abdominal trauma $(25,26)$. 
The most common complications encountered in patients undergoing splenectomy due to trauma or liver repair are wound infection, pneumonia, sepsis, and ARDS. Non-operative follow-up of patients with blunt abdominal trauma reduces the duration of hospital stay as well as protecting the patient from the possibility of postoperative complications $(27,28)$. In this study, the duration of hospitalization of the patients who were followed-up without surgery was found to be significantly lower. On the other hand, the most common complications are bilioma, pseudoaneurysm and intraabdominal abscess in patients with non-operative follow-up $(29,30)$. In this study, no complication was detected in the patients without surgery who were followed up, and 4 of the operated patients developed an infection

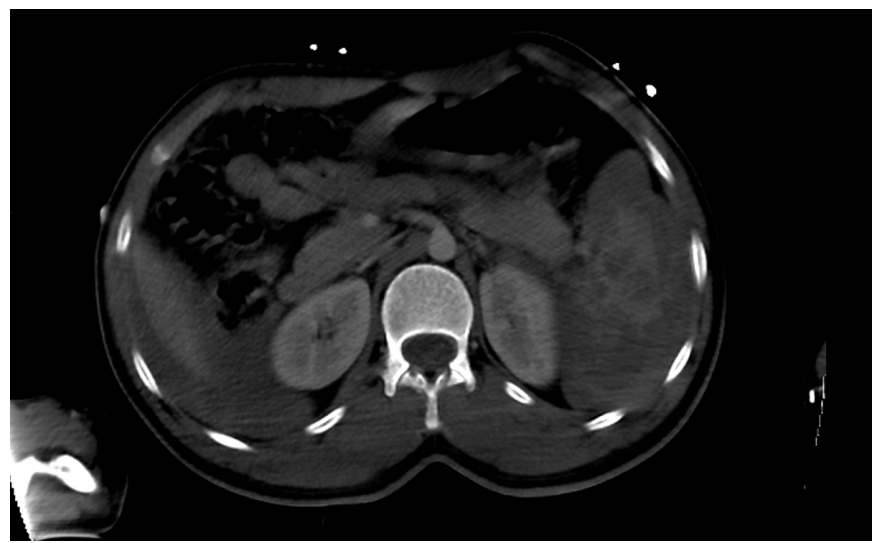

Figure 1. Grade IV spleen laceration

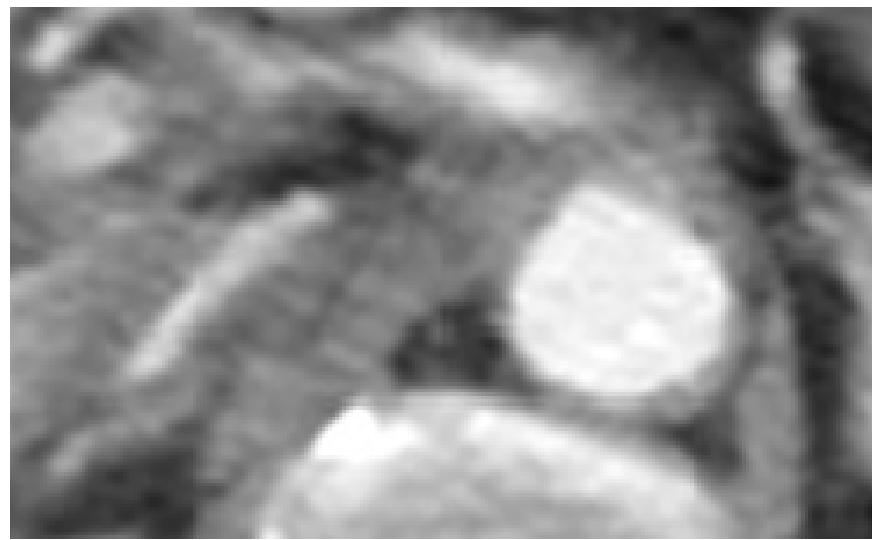

Figure 2. Liver laceration

\section{CONCLUSION}

The present study showed that low-grade liver and spleen injuries can be successfully followed up without surgery. On the other hand, non-operative follow-up has its own complications. The days of hospitalization and the time of return to work are shorter in non-surgical follow-up. These complications should be taken care of, additional interventions should be performed if it develops.

\section{ETHICAL DECLARATIONS}

Ethics Committee Approval: The studywas approved by the Hitit University Medical Faculty Erol Olçok Training and Research Hospital Non-interventional Research Ethics Committee (Date: 26.10.2018, Decision No: 2018180).

Referee Evaluation Process: Externally peer-reviewed.

Conflict of Interest Statement: The authors have no conflicts of interest to declare.

Financial Disclosure: The authors declared that this study has received no financial support.

Author Contributions: All of the authors declare that they have all participated in the design, execution, and analysis of the paper, and that they have approved the final version.

\section{REFERENCES}

1. Smith SR, Morris L, Spreadborough S, et al. Management of blunt splenic injury in a UK major trauma centre and predicting the failure of non-operative management: a retrospective, crosssectional study. Eur J Trauma Emerg Surg 2018; 44: 397-406.

2. Coccolini F, Montori G, Catena F, et al. splenic trauma: WSES classification and guidelines for adult and pediatric patients. World J Emerg Surg 2017; 12: 40.

3. Burlew CC, Moore EE. Trauma. In: Brunicardi FC, Andersan DK, Biliar TR, et al (eds). Schwartz's Principles of Surgery. United State of America, McGraw-Hill Medical Publishing, 2015, p: 129-88.

4. Afifi I, Abayazeed S, El-Menyar A, et al. Blunt liver trauma: a descriptive analysis from a level I trauma center. BMC Surg 2018; 18: 42 .

5. Yanar H, Ertekin C, Taviloglu K, et al. Non-operative treatment of multiple intra-abdominal solid organ injury after blunt abdominal trauma. J Trauma 2008; 64: 943-8.

6. Boese CK, Hackl M, Muller LP, et al. Non-operative management of blunt hepatic trauma: A systematic review. J Trauma Acute Care Surg 2015; 79: 654-60.

7. Walker ML. Nonoperative management of the damaged spleen: a community surgeon looks back. Am Surg 2017; 83: 502-6.

8. Brady RR, Bandari M, Kerssens JJ, et al. Splenic trauma in Scotland: demographics and outcomes. World J Surg 2007; 31: 2111-6.

9. Okus A, Sevinc B, Ay S, et al. Conservative management of abdominal injuries. Ulus Cerrahi Derg 2013; 29: 153-7.

10. Van der Wilden GM, Velmahos GC, Emhoff T, et al. Successful non-operative management of the most severe blunt liver injuries: a multicenter study of the research consortium of new England centers for trauma. Arch Surg 2012; 147: 423-8.

11. Hassan R, Abd Aziz A, Md Ralib AR, et al. Computed tomography of blunt spleen injury: a pictorial review. Malays J Med Sci. 2011 Jan; 18: 60-7.

12. Federle MP, Courcoulas AP, Powell M, et al. Blunt splenic injury in adults: clinical and CT criteria for management, with emphasis on active extravasation. Radiology 1998; 206: 137-42.

13. Kokabi N, Shuaib W, Xing M, et al. Intra-abdominal solid organ injuries: an enhanced management algorithm. Can Assoc Radiol J 2014; 65: 301-9. 
14. Yiannoullou P, Hall C, Newton $\mathrm{K}$, et al. A review of the management of blunt splenic trauma in England and Wales: have regional trauma networks influenced management strategies and outcomes? Ann R Coll Surg Engl 2017; 99: 63-9.

15. Oh JS, Mentzer CJ, Abuzeid AM, et al. Delayed Splenic Rupture with Normal Admission CT Scan after Blunt Trauma. Am Surg 2016; 82: e223-4.

16. Riezzo I, Di Battista B, De Salvia A, et al. Delayed splenic rupture: dating the sub-capsular hemorrhage as a useful task to evaluate causal relationships with trauma. Forensic Sci Int 2014; 234: 6471.

17.Stassen NA, Bhullar I, Cheng JD, et al. Selective non-operative management of blunt splenic injury: an eastern association for the surgery of trauma practice management guideline. J Trauma 2012; 73: 294-300.

18. Parks NA, Davis JW, Forman D, et al. Observation for nonoperative management of blunt liver injuries: how long is long enough? J Trauma 2011; 70: 626-9.

19. Suen K, Skandarajah AR, Knowles B, et al. Changes in the management of liver trauma leading to reduced mortality: 15year experience in a major trauma centre. ANZ J Surg 2016; 86: 894-9.

20. Gaarder C, Gaski IA, Næss PA. Spleen and liver injuries: when to operate. Curr Opin Crit Care 2017; 23: 520-6.

21. Kim KH, Kim JS, Kim WW. Outcome of children with blunt liver or spleen injuries: experience from a single institution in Korea. Int J Surg 2017; 38: 105-108.

22. Kong VY, Jeetoo D, Naidoo LC, et al. Isolated free intra-abdominal fluid on CT in blunt trauma: The continued diagnostic dilemma. Chin J Traumatol 2015; 18: 357-9.

23. Sharma OP, Oswanski MF, Singer D, et al. Assessment of nonoperative management of blunt spleen and liver trauma. Am Surg 2005; 71: 379-86.

24. Olthof DC, Joosse P, van der Vlies CH, et al. Prognostic factors for the failure of non-operative management in adults with blunt splenic injury. J Trauma 2013; 74: 546-57.

25. Melikian R, Goldberg S, Strife BJ, et al. Comparison of MDCT protocols in trauma patients with suspected splenic injury: superior results with protocol that includes arterial and portal venous phase imaging. Diagn Interv Radiol 2016; 22: 395-9.

26. Tugnoli G, Bianchi E, Biscardi A, et al. Non-operative management of blunt splenic injury in adults: there is (still) a long way to go. The results of the Bologna-Maggiore hospital trauma center experience and development of a clinical algorithm. Surg Today 2015; 45: 1210-7.

27. Norrman G, Tingstedt B, Ekelund $M$, et al. Non-operative management of blunt liver trauma: feasible and safe also in centres with a low trauma incidence. HPB (Oxford) 2009; 11: 50-6.

28. Alamri Y, Moon D, Yen DA, et al. Ten-year experience of splenic trauma in New Zealand: the rise of non-operative management. N Z Med J 2017; 130: 11-8.

29. Mehta N, Babu S, Venugopal K. An experience with blunt abdominal trauma: evaluation, management and outcome. Clin Pract. 2014; 4: 599.

30.El-Matbouly M, Jabbour G, El-Menyar A, et al. Blunt splenic trauma: assessment, management and outcomes. Surgeon 2016; 14: 52-8. 